\title{
$f$-Best Coapproximation in Quotient Topological Vector Spaces
}

\author{
Majid Abrishami-Moghaddam
}

Department of Mathematics, Birjand Branch, Islamic Azad University, Birjand, Iran; m.abrishami.m@gmail.com

\begin{abstract}
In this paper we give new results on the best coapproximation in the Hausdorff topological vector space $X$. Assume that $f$ be a real valued function on $X$ and we present some results regarding $f$-best coapproximation. We determine under what conditions $f$-coproximinality can be transmitted to the quotient spaces and conversely.

2010 Mathematics Subject Classification: 41A52, 57N17.
\end{abstract}

Keywords: f-best Coapproximation, f-coChebyshev, f-compact, f-quasi-coChebychev

\section{Introduction}

The notion of $f$-best approximation in a vector space $X$ was given by Breckner and Brosowski ${ }^{2}$ A few years later, T. D. Narang introduced the notions of $f$-best approximation and $f$-best coapproximation in a Hausdorff topological space $^{3,4}$. Recently the author in1 obtained some properties of $f$-best approximation sets in quotient spaces. In this paper we develop the theory of $f$-best coapproximation in quotient topological vector spaces. We want to determine under what conditions $f$-coproximinality can be transmitted to the quotient spaces and conversely.

Let $X$ be a Hausdorff topological vector space over $\mathbb{R}$ and $f$ a real valued function. Let $K$ be a nonempty closed subset of $X$ and $x \in X$. Element $k_{0} \in K$ is said to be an $f$-best approximation to $x$ in $K$ if

$$
f\left(x-k_{0}\right)=\inf \{f(x-k): k \in K\} .
$$

Furthermore, element $k_{0} \in K$ is said to be an $f$-best coapproximation to $x$ in $K$ if

$$
f\left(k-k_{0}\right) \leq f(x-k)
$$

for all $k \in K$. We denote by $R_{K}^{f}(x)$ the collection of all such $k_{0} \in K$. The set $K$ is said to be $f$-coproximinal if $R_{K}^{f}(x)$ is nonempty for each $x \in X$, and $f$-coChebysev if $R_{K}^{f}(x)$ is exactly singleton for each $x \in X$.
Putting

$\breve{K}^{f}=\{x \in X: f(k) \leq f(x-k)$ for all $k \in K\}=\left(R_{K}^{f}\right)^{-1}(\{0\})$.

It is clear that $k_{0} \in R_{K}^{f}(x)$ if and only if $x-k_{0} \in \breve{K}^{f}$.

A subset $K$ of $X$ is called $f$-compact if every sequence $\left\{k_{n}\right\}$ in $K$ has a subsequence $\left\{k_{n_{i}}\right\}$ of $\left\{k_{n}\right\}$ and $k_{0} \in K$ such that $f\left(k_{n_{i}-} k_{0}\right) \rightarrow 0 . K$ is called $f$-quasi-coChebyshev if $R_{K}^{f}(x)$ is nonempty and $f$-compact set in $X$ for every $x \in X$.

A function $f: X \rightarrow \mathbb{R}$ is absolutely homogeneous if $f(\alpha x)=|\alpha| f(x)$ for all $\alpha \in \mathbb{R}$ and $x \in X$.

When $X$ is a normed linear space over the filed of real numbers and $f(x, y)=\|x-y\|$ for all $x, y \in X$, the notions introduced above coincide with the corresponding notions that already exists in literature.

\section{Set of $f$-Coapproximations}

In this section, we give some characterizations of $f$-coproximinal sets in $X$.

\section{THEOREM 1}

Let $X$ be a topological vector space and $f$ be a real valued function. 
If $K$ is a subset of $X$, then

1) $R_{(K+y)}^{f}(x+y)=R_{K}^{f}(x)+y$ for all $x, y \in X$.

2) $K$ is $f$-coproximinal ( $f$-coChebyshev) if and only if $K+y$ is $f$-coproximinal ( $f$-coChebyshev) for every $y \in X$. Furthermore if $f$ is absolutely homogeneous, then

3) $R_{\alpha K}^{f}(\alpha x)=|\alpha| R_{K}^{f}(x$ for all $x \in X$ and $\alpha \in \mathbb{R}$.

4) $K$ is $f$-coproximinal ( $f$-coChebyshev) if and only if $\alpha K$ is $f$-coproxinminal ( $f$-coChebyshev) for all $\alpha \in \mathbb{R}$.

\section{Proof:}

(1) $k_{0}+y \in R_{K+y}^{f}(x+y)$ if and only if $f\left(k_{0}+y-(k+y)\right) \leq$ $f(x+y-(k+y))$ for all $k \in K$, if and only if $f\left(k_{0}-k\right) \leq$ $f(x-k)$ for all $k \in K$, if and only if $k_{0} \in R_{K}^{f}(x)$. Thus $R_{K+y}^{f}(x+y)=R_{K}^{f}(x)+y$.

(2) It is clear by (2).

(3) If $\alpha=0$, the result is true. Thus assume that $\alpha \neq 0$. $k_{0} \in R_{\alpha K}^{f}(\alpha x)$ if and only if $k_{0} \in \alpha K$ and $f\left(k_{0}-\alpha k\right) \leq$ $f(\alpha x-\alpha k)$ for all $k \in K$, if and only if $\frac{1}{\alpha} k_{0} \in K$ and $|\alpha| f\left(\frac{1}{\alpha} k_{0}-k\right) \leq|\alpha| f(x-k)$ for all $k \in K$, and this implies that $\frac{1}{\alpha} k_{0} \in R_{K}^{f}(x)$. So $k_{0} \in \alpha R_{K}^{f}(x)$.

(4) It is clear by (3).

\section{Corollary 1}

Let $X$ be a topological vector space and $f$ be a real valued function. If $M$ be a nonempty subspace of $X$, then

(i) $R_{M}^{f}(x+y)=R_{M}^{f}(x)+y$, for every $x, y \in X$.

(ii) $R_{M}^{\alpha f}(\alpha x)=\alpha R_{M}^{f}(x)$ for every $x \in X$ and $\alpha \in \mathbb{R}\{0\}$.

\section{ProOf:}

The proof is an immediate consequence of theorem(2.1) and this fact that $M+y=M$ and $\alpha M=M$ for all $y \in M$ and $\alpha \in \mathbb{R}\{0\}$.

\section{THEOREM 2}

If $K$ is a subspace of $X$ and $f$ is e real function, then

(i) $K$ is $f$-coproximinal if and only if $X=K+\breve{K}^{f}$.

(ii) $K$ is a $f$-coChebyshev subspace if and only if $X=K \oplus \breve{K}^{f}$.

\section{ProOf:}

(i) $(\Rightarrow)$ Assume that $K$ is $f$-coproximinal, $x \in X$ and $k_{0} \in R_{K}^{f}(x)$. Then, $x-k_{0} \in \breve{K}^{f}$. Now, $x=k_{0}+\left(x-k_{0}\right) \in$ $K+\breve{K}^{f}$. Hence $X=K+\breve{K}^{f}$.

$(\Leftarrow)$ Let $x \in X=K+\breve{K}^{f}$.Then $x=k_{0}+\breve{k}, k_{0} \in K, \breve{k} \in \breve{K}^{f}$ and so $0 \in R_{K}^{f}(\breve{k})=R_{K}^{f}\left(x-k_{0}\right)=R_{K}^{f}(x)-k_{0}$; $\quad$ hence $k_{0} \in R_{K}^{f}(x)$. Therefore $K$ is $f$-coproximinal.

(ii) $(\Rightarrow)$ Soppose that $K$ is $t$-coChebyshev subspace, $x \in X$, and $x=k_{1}+\breve{k}_{1}=k_{2}+\breve{k}_{2}$, where $k_{1}, k_{2} \in K$ and $\breve{k}_{1}, \breve{k}_{2} \in \breve{K}^{f}$.

We show that $k_{1}=k_{2}$ and $\breve{k}_{1}=\breve{k}_{2}$. Since $x=k_{1}+\breve{k}_{1}=$ $k_{2}+\breve{k}_{2}$, then $x-k_{1}=\breve{k}_{1}, x-k_{2}=\breve{k}_{2}$, this implies that $k_{1}, k_{2} \in R_{K}^{f}(x)$. Therefore $k_{1}=k_{2}$, it follows that $\breve{k}_{1}=\breve{k}_{2}$. Thus $X=K \oplus \breve{K}^{f}$.

$(\Leftarrow)$ Let $X=K \oplus \breve{K}^{f}$, and suppose for $x \in X$, there exist $k_{1}, k_{2} \in R_{K}^{f}(x)$. Then $x-k_{1}, x-k_{2} \in \breve{K}^{f}$, and therefore, $x=k_{1}+\breve{k}_{1}=k_{2}+\breve{k}_{2}$, where $\breve{k}_{1}=x-k_{1}, \breve{k}_{2}=x-k_{2}$, It follows $k_{1}=k_{2}$ and $\breve{k}_{1}=\breve{k}_{2}$.

\section{EXAMPLE 1}

Let $X=\mathbb{R}^{2}$ and $K=\{(x, y): x=y\}$, consider $f(x, y)=x^{2}+y^{2}$, then $\breve{K}^{f}=\{(x, y): x=-y\}$, and $R_{K}^{f}(a, b)=\left(\frac{a+b}{2}, \frac{a+b}{2}\right)$

Therefore $K$ is $f$-coChebyshev.

\section{3. f-Coapproximation in Quotient Space}

Let $X$ be a topological vector space and $M$ be a closed subspace of $X$, and $f: X \rightarrow \mathbb{R}$ be a symmetric function (i.e., $f(-x)=f(x)$. Define

$$
\tilde{f}(x+M)=\inf \{f(x+y) \mid y \in M\} .
$$

\section{THEOREM 3}

Let $M$ be a closed subspace of $X$, and $K \supseteq M$ an $f$-coproximinal subspace of $X$. If $k_{0} \in R_{K}^{f}(x)$, then $k_{0}+M \in R_{\frac{K}{M}}^{\tilde{f}}(x+M)$. 


\section{ProOF:}

Assume that $k_{0} \in R_{K}^{f}(x)$ and $k_{0}+M$ not in $R_{\frac{K}{M}}^{\tilde{f}}(x+M)$. Then there exists $k^{\prime} \in K$ such that

$$
\tilde{f}\left((x+M)-\left(k^{\prime}+M\right)\right)<\tilde{f}\left(\left(k^{\prime}+M\right)-\left(k_{0}+M\right)\right) .
$$

That is

$$
\tilde{f}\left(\left(x-k^{\prime}\right)+M\right)<\tilde{f}\left(\left(k^{\prime}-k_{0}\right)+M\right) .
$$

Hence there exists $m \in M$ such that

$$
f\left(x-k^{\prime}-m, t\right)<f\left(k^{\prime}-k_{0}+m\right) .
$$

Thus,

$$
f\left(x-\left(k^{\prime}+m\right)\right)<f\left(\left(k^{\prime}+m\right)-k_{0}\right) .
$$

Therefore $k_{0}$ is not a $f$-best coapproximation to $x$ from $K$; which is a contradiction. So we have $k_{0}+M \in R_{\frac{K}{M}}^{\tilde{f}}(x+M)$ and the proof is completed.

\section{Corollary 2}

Let $M$ be a closed subspace of $X$ and $K$ an $f$-coproximinal subspace of $X$ containing $M$. Then $\frac{K}{M}$ if an $\tilde{f}$-coproximinal subspace of $\frac{X}{M}$

\section{Corollary 3}

Let $M$ be a closed subspace of $X$ and $K$ an $f$-coproximinal subspace of $X$ containing $M$. Then

$$
\pi\left(R_{K}^{f}(x)\right) \subseteq R_{\frac{K}{M}}^{f}(x+M) .
$$

\section{ThEOREM 4}

Let $M$ be a f-proximinal closed subspace of $X$ and $K$ a subspace of $X$ containing $M$. If $k_{0}+M \in R_{K}^{\tilde{f}}(x+M)$, then there exists $m_{0} \in M$ such that $k_{0}+m_{0} \in R_{K}^{f}(x)$.

\section{Proof:}

Let $k_{0}+M \in R_{K}^{\tilde{f}}(x+M)$ where $k_{0} \in K$. Then for every $k \in K$,

$$
\tilde{f}\left((k-M)-\left(k_{0}-M\right)\right) \leq \tilde{f}((x-M)-(k-M)),
$$

or

$$
\left.\left.\tilde{f}\left(\left(k-k_{0}\right)+M\right)\right) \leq \tilde{f}((x-k)+M)\right) .
$$

By $f$-proximinality of $K$ in $M$, there exists $m_{0} \in M$ such that

$$
f\left(\left(k-k_{0}\right)-m_{0}\right)=\tilde{f}\left(\left(k-k_{0}\right)+M\right) .
$$

Now we have

$$
\begin{aligned}
f\left(\left(k-\left(k_{0}+m_{0}\right)\right)\right. & \left.=f\left(\left(k-k_{0}\right)-m_{0}\right)\right) \\
& \left.=\tilde{f}\left(\left(k-k_{0}\right)+M\right)\right) \\
& \leq \tilde{f}((x-k)+M) \\
& \leq f(x-k)
\end{aligned}
$$

for every $k \in K$. Therefore $k_{0}+m_{0} \in R_{K}^{f}(x)$.

\section{Corollary 4}

Let $M$ be a f-proximinal closed subspace of $X$ and $K \supseteq M$ a subspace of $X$. If $\frac{K}{M}$ is $\tilde{f}$-coproximinal in $\frac{X}{M}$, then $K$ is t-coproximinal in $X$.

\section{Corollary 5}

Let $M$ be a f-proximinal closed subspace of $X$ and $K \supseteq M$ a subspace of $X$, then

$$
\pi\left(R_{K}^{f}(x)\right)=R_{\frac{K}{M}}^{f}(x+M)
$$

\section{Proof:}

By corollary (3.3) we obtain

$$
\pi\left(R_{K}^{f}(x)\right) \subseteq R_{\frac{K}{M}}^{f}(x+M)
$$

Also by theorem (3.1), $\frac{K}{M}$ is $\tilde{f}$-coproximinal in $\frac{X}{M}$. Now let $k_{0}+M \in R_{\frac{K}{M}}^{\tilde{f}}(x+M)$, where $k_{0} \in K$. Now by theorem (3.4) $K$ is $f$-coproximinal and there exists $m_{0} \in M$ 
such that $k_{0}+m_{0} \in R_{K}^{f}(x)$, and so $k_{0}+M \in \pi\left(R_{K}^{f}(x)\right)$;

hence $R_{\frac{K}{M}}^{\tilde{f}}(x+M) \subseteq \pi\left(R_{K}^{f}(x)\right)$, and the proof is complete.

\section{TheOREM 5}

Let $M$ be a f-proximinal closed subspace of $X$ and $K \supseteq M$ a subspace of $X$. If $K$ is $f$-coChebyshev then $\frac{K}{M}$ is a $\tilde{f}$ coChebyshev subspace of $\frac{X}{M}$.

\section{Proof:}

By theorem (3.1), $\frac{K}{M} \tilde{f}$-is coproximinal. Let $x+M \in$ $(X / M)(K / M)$ be arbitrary and $k_{1}+M, k_{2}+M \in R_{\frac{W}{M}}^{\tilde{f}}(x+M)$. By theorem (3.4) there exists $m_{1}, m_{2} \in M$ such that $k_{1}+m_{1}$, $k_{2}+m_{2} \in R_{k}^{f}(x)$. Since $K$ is $f$-coChebyshev $k_{1}+m_{1}=k_{2}+m_{2}$ and then $k_{1}+M=k_{2}+M$.

\section{LEMMA 1}

Let $M$ be a f-proximinal closed subspace of $X$ and $K \supseteq M a$ subspace of X. If $\breve{K}^{f}$ is convex, then $\left(\frac{K}{M}\right)^{\tilde{f}}=\left(\begin{array}{c}\frac{\tilde{f}}{M} \\ )^{-1}\end{array}\right.$

is convex.

\section{ProOF:}

Let $\quad x+M, y+M \in\left(\frac{\tilde{K}}{M}\right)^{\tilde{f}}$ and $\quad 0<\lambda<1 . \quad$ Then $M \in R_{\frac{K}{M}}^{\tilde{f}}(x+M)$ and $M \in R_{\frac{K}{M}}^{\tilde{f}}(y+M)$. Now from corollary (3.6) there exists $k_{1} \in R_{K}^{f}(x)$ and $k_{2} \in R_{K}^{f}(x)$ such that $\pi\left(k_{1}\right)=\pi\left(k_{2}\right)=M$. Therefore $x-k_{1}, y-k_{2} \in \breve{K}^{f}$. Since $\breve{K}^{f}$ is convex then $\lambda\left(x-k_{1}\right)+(1-\lambda)\left(y-k_{2}\right) \in \breve{K}^{f}$. It follows that $\lambda k_{1}+(1-\lambda) k_{2} \in R_{K}^{f}(\lambda x+(1-\lambda) y)$; also

$$
\begin{aligned}
\pi\left(\lambda k_{1}+(1-\lambda) k_{2}\right) & =\lambda \pi\left(k_{1}\right)+(1-\lambda) \pi\left(k_{2}\right) \\
& =\lambda M+(1-\lambda) M \\
& =M .
\end{aligned}
$$

Now by corollary (3.6), $M \in R_{\frac{K}{M}}^{\tilde{f}}(\lambda x+(1-\lambda) y+M)$; that is $\lambda\left(x-k_{1}\right)+(1-\lambda)\left(y-k_{2}\right) \in\left(\frac{\tilde{K}}{M}\right)^{\tilde{f}}$. Therefore $\left(\frac{\tilde{K}}{M}\right)^{\tilde{f}}$ is convex.

\section{TheOREM 6}

Let $M$ be a f-proximinal closed subspace of $X$ and $K \supseteq M$ be a f-coproximinal subspace of $X$ such that $K$ is $f$-quasicoChebyshev. Then $\frac{K}{M}$ is $\tilde{f}$-quasi-coChebyshev.

\section{Proof:}

Since $K$ is $f$-coproximinal, therefore $\frac{K}{M}$ is $f$-coproximinal. Let $x+M \in \frac{X}{M}$ and $\left\{k_{n}+M\right\}$ be an arbitrary sequence in $R_{\frac{K}{M}}^{\tilde{f}}(x+M)$. Then for every $k+M \in \frac{K}{M}$,

$$
\begin{aligned}
\tilde{f}\left(\left(k_{n}-k\right)+M\right) & =\tilde{f}\left(\left(k_{n}+M\right)-(k+M)\right) \\
& \leq \tilde{f}((x+M)-(k+M)) \\
& =\tilde{f}((x-k)+M) \\
& \leq \tilde{f}(x-k) .
\end{aligned}
$$

Since $M$ is $f$-proximinal, by theorem (3.4) there exist $m_{n} \in M$ such that $k_{n}+m_{n} \in R_{K}^{f}(x)$, for all $n \geq 1$. Now since $R_{K}^{f}(x)$ is $f$-compact, there exists a subsequence $\left\{k_{n_{i}}+m_{n_{i}}\right\}$ such that it is $f$-converges to an element $\alpha_{0} \in R_{K}^{f}(x)$; therefore $\alpha_{0}+M \in R_{\frac{K}{M}}^{\tilde{f}}(x+M)$. It follows that $\left\{k_{n_{k_{i}}}+M\right\}$ is $f$-converges to an element $\alpha_{0}+M$. Hence $\frac{K}{M}$ is quasi $\tilde{f}$-coChebyshev.

\section{References}

1. Moghaddam MA. On $f$-best approximation in quotient topological vector spaces. International Mathematical Forum. 2010; 5(12):587-95.

2. Breckner WW, Brosowski B. Ein kriterium zur charakterisierung von sonnen. Mathematika. 1971; 13:181-8.

3. Narang TD. On $f$-best approximation in topological spaces. Arch Math. 1985; 21(4):229-34.

4. Narang TD. Approximation relative to an ultra function. Arch Math. 1986; 22(4):181-6. 